\title{
Analysis on Semi Active Control of Eccentric Structure with Torsion Response by a TMD and MR Damper

\author{
Qu Chengzhong, a and Ma Jiayou, ${ }^{1, b}$
} \\ ${ }^{1}$ Northeast Dianli University, Institute of civil engineering, Jilin, China \\ aquchegnzhong@126.com, bandie0614@163.com
}

Keywords: Intelligent control Method, Magneto-rheological Fluid Damper, Seismic torsion.

\begin{abstract}
In this paper, we studied the control effect of irregular structures with translation-torsion coupling response under earthquake loading by using semi-active control method. On the base of traditional resistance design method, we couple magneto-rheological fluid damper (MR) and tuned mass dampers (TMD) to controlling device, applying semi-active control strategy to control the torsion response. We build MR-TMD coupling model of semi-active control algorithm and simulation of processes, then study the torsion resistance problems.
\end{abstract}

\section{Introduction}

Overstep torsional effect can lead to the degradation of the seismic performance of the structure, and accelerate the destruction of the structure. The traditional seismic design method can not solve the problem of torsional vibration of eccentric structure under earthquake action. This paper is intended to study the adjustment of the control device and reduce the torsion of the structure without changing the cross section of the structure. In this paper, the design proposal is provided for the control field of the eccentric structure with a tuned mass damper (TMD) and a magneto rheological(MR) damper. The study of magneto rheological technology is mainly based on the application of magneto rheological fluid, and the intelligent vibration control system formed by the combination of the magnetic rheological fluid and structural components is promising. Analysis the control effect of MR-TMD damping system to structure by installing MR dampers to change and make up for deficiencies in TMD in control alone. Taking a typical eccentric structure workshop for the study of vibration control object, the semi active control measures MR-TMD study the structure vibration response. This topic has very good theoretical value and engineering applications.

\section{Eccentric structure analysis}

Basic Assumption of Eccentric Structure. Eccentric structure, whose center of stiffness digresses from center of mass, in addition to generating translational vibration will also produce torsional vibration. If ignore the floor bending or shear deformation, floor can only arouse translation and rotation. At this time with a floor on the translation and rotation are the same. Based on the above analysis, the following assumptions are adopted in the analysis model in the calculation process:

A. Assume the building floor is rigid floor, in a plane infinite stiffness and plane bending stiffness is negligible;

B. structure of floor mass and moment of inertia in floor place, structure considering the torsion effect on each floor with 3 degrees of freedom;

C. considering only the horizontal components of earthquakes, regardless of reversing or swing components;

D. the bottom of the structure and foundation are absolutely consolidated, regardless of interaction between superstructure and base and foundation.

Simplified Calculation Model of Eccentric Structure. Based on simplifying assumptions made in the previous section, analysis of eccentric structure of translation-torsion coupling seismic response, series can be used as a simplified model. 

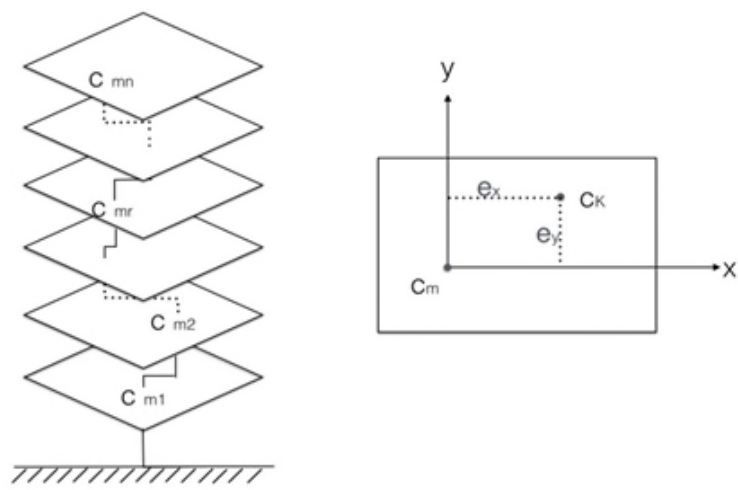

Fig. 1 Simplified model of eccentric structure.

Due to the structure of the center of mass and stiffness centers often do not coincide, the structure under seismic action except in translation, but there will be reversed, namely life-torsional coupled vibration response. In order to reduce the structural seismic response, except by increasing the structure stiffness, strength and ductility of traditional seismic methods, can also control device is to structure to consumption, vibration energy absorbing structure. This paper analyses vibration of an existing high-rise eccentric structure, because of the unique function of the structure requirements, its architectural form and component layout is limited, resulting in reverse in its vibration is inevitable. Obviously for the seismic design of high-rise plant is very unfavorable.

Coupling Dynamic Equation of Eccentric Structure. Spectacular series rigid model, would have $3 n$ degrees of freedom for $n$-tier architecture, $3 n$ equations can be established, the coordinate origin at the point in the center of each floor. On the ground under the action of $\mathrm{x}$ and $\mathrm{y}$, bi-directional moving components, translational torsion coupling series rigid equation of motion is:

$$
\begin{aligned}
& M \ddot{u}+C \dot{u}+K u=M I \ddot{u}_{g} \\
& \begin{array}{l}
u=\left[u_{x}^{T}, u_{y}^{T}, u_{\theta}^{T}\right]^{T} \\
u_{x}=\left[u_{1 x}, u_{2 x}, \ldots, u_{n x}\right]^{T}, u_{y}=\left[u_{1 y}, u_{2 y}, \ldots, u_{n y}\right]^{T}, u_{\theta}=\left[u_{1 \theta}, u_{2 \theta}, \ldots, u_{n \theta}\right]^{T}
\end{array}
\end{aligned}
$$

\section{Semi-active Control}

Intelligent material is a new kind of material which has the function of sensing and driving at the same time. Controllable fluid damper is a kind of stable and reliable vibration reduction system [1]. The runny nose controllable damper damping characteristics can be changed by adjusting the electric field or magnetic field, and its installation in the structure the structure control for semi active control.

This paper used simplified Bingham model (Fig. 2) to simulate dynamic force of MR damper.

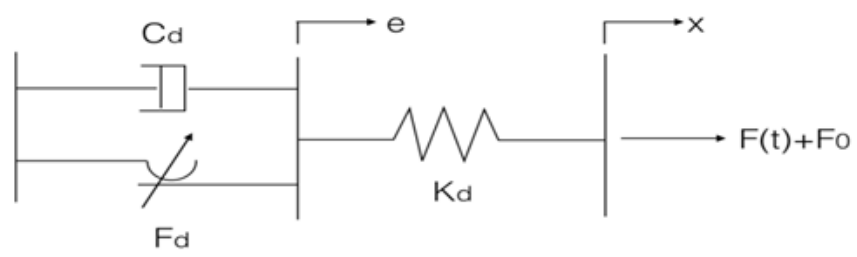

Fig. 2 Simplified Bingham model of MR.

Due to actual in the MR damper can provide damping force is not up to the control force control law, hypothesis $\mathrm{F}_{\min }, \mathrm{F}_{\max }$ respectively for MR damper can provide minimum and maximum damping force, using linear quadratic optimal control (LQR) algorithm is used to obtain the optimal control force is the mark [2]. In this paper, the following control strategy is determined, the actual force applied to the coupling system and the corresponding control strategy: 


$$
F(t)=\left\{\begin{array}{cc}
F_{\text {min }} \operatorname{sgn}(U) & |U|<F_{\min } \\
U & F_{\min }<|U|<F_{\max } \\
F_{\text {max }} \operatorname{sgn}(U) & |U|>F_{\text {max }}
\end{array}\right.
$$

TMD control process does not depend on the control of structural response and interference information, but traditional tuning quality system there are still some problems, mainly reflected in multi modal damping effect still needs to be improved, multidimensional vibration analysis method still need to be improved and so on.

In the vicinity of the center of mass of the TMD structure, the tuned mass block is connected with the limit baffle by a horizontal viscous damper with an appropriate stiffness to ensure that the tuned mass block can quickly and repeatedly vibrate.

When the structure under the action of earthquake vibration, level to the tuned mass part movement, tuned mass blocks based on and the structure of relative deformation produced by the horizontal inertial force[3] and MR damper damping force to produce reaction to the structure to reduce the vibration amplitude of the structure in the horizontal direction.

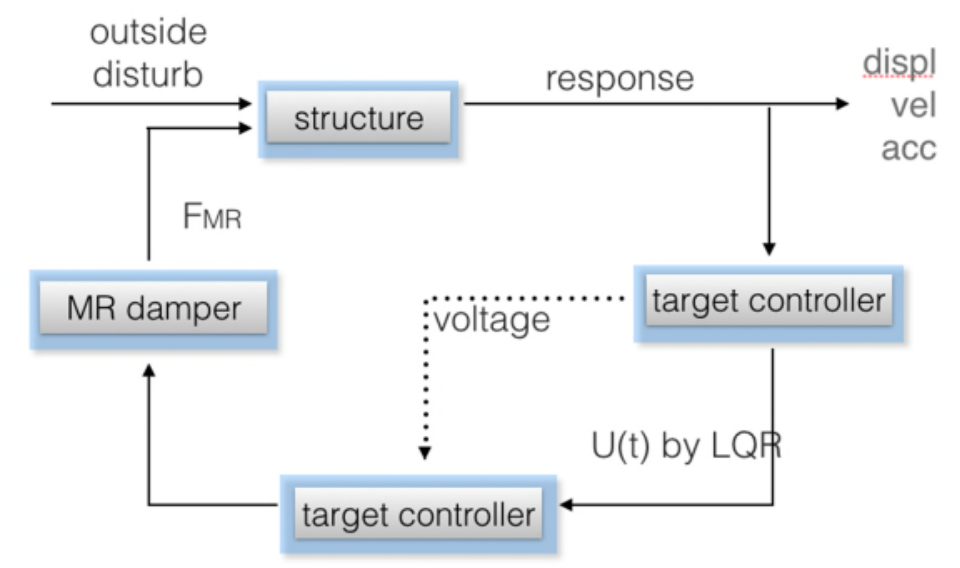

Fig. 3 flow chart of Semi control by MATLAB.

The semi-active control algorithm (Fig. 3) is based on the structure of the information parameters (stiffness matrix, mass matrix, damping matrix) to establish the dynamic equation of the structure according to the Darren Bell principle. And set up a state variable Z, and the establishment of the state equation of structure, by the equation of state in some active control algorithms, such as LQR and LQG method [4] we calculate the optimal damper control force vector of the high-rise plant state equation using the classical linear quadratic linear (LQR) optimal control algorithm for optimal control and semi active control strategy, combined with the proposed MR damper model and the external control, reached the semi active control output function in building structure, reciprocating cycle of the whole process constitute half of active control strategy.

$$
\begin{aligned}
& M \ddot{u}+C \dot{u}+K u=-M I \ddot{u}_{g}+f_{T M D}+I_{s} f_{s}(t) \\
& m_{d} \ddot{u}_{d}+f_{s}(t)+k_{d}\left(u_{d}-u_{N}\right)=-m_{d} \ddot{u}_{g}
\end{aligned}
$$

\section{Simulate Seismic Torsion Response}

For eccentric structure, due to stiffness center partial in side, mass and stiffness center not coincide, even in horizontal seismic action, also will provoked reverse vibration, away from just center of stiffness smaller of component due to side moved volume of increased [5], its by of earthquake role also will significantly increases, beyond allows anti-force and deformation limit and occurred damage, even led to whole structure for side component failure and collapsed. 


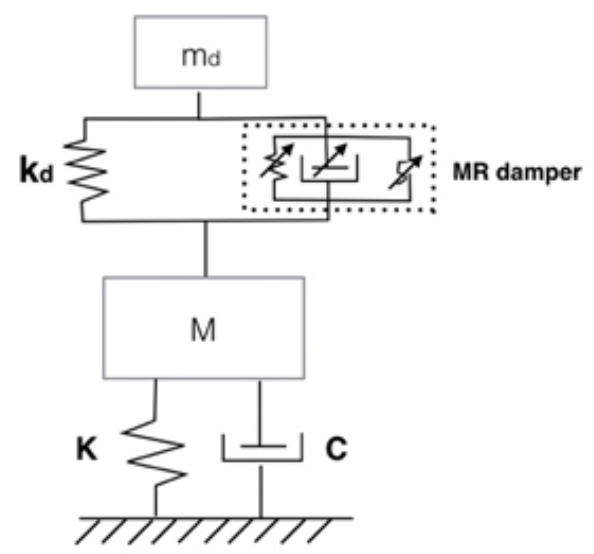

Fig. 4 Model of structure with TMD-MR.

Main structure mass is $1250 \mathrm{t}$, stiffness is $4.28 \times 105 \mathrm{~N} / \mathrm{m}$ hypothesis subjected to El Centro (NS) seismic waves, seismic wave acceleration scroll $40 \mathrm{gal}$, MR damper for the convenience of in this paper, we assume that the maximum damping force, minimum force respectively is $800 \mathrm{KN}$ and $100 \mathrm{kN}$. With this control strategy, the simulation calculation structure is obtained by MR-TMD control system and the control system effect comparison is shown in the flowing figures.

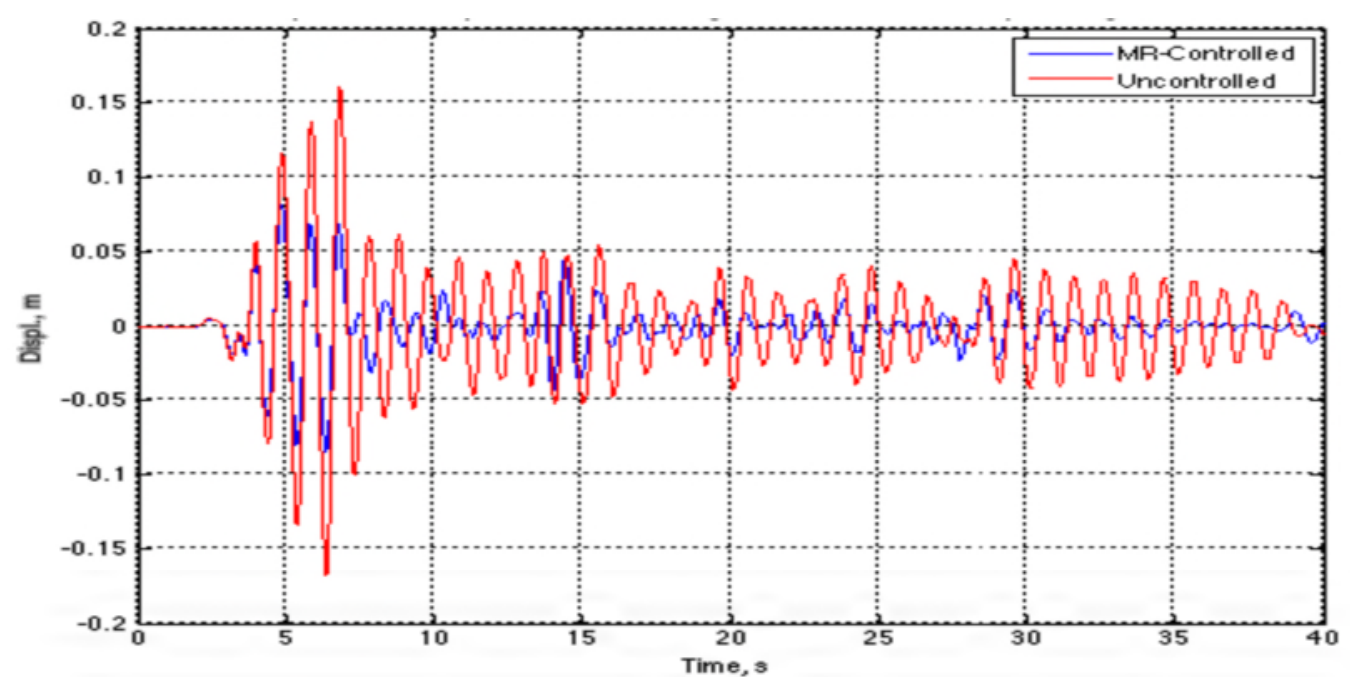

Fig. 5 Displacement response.

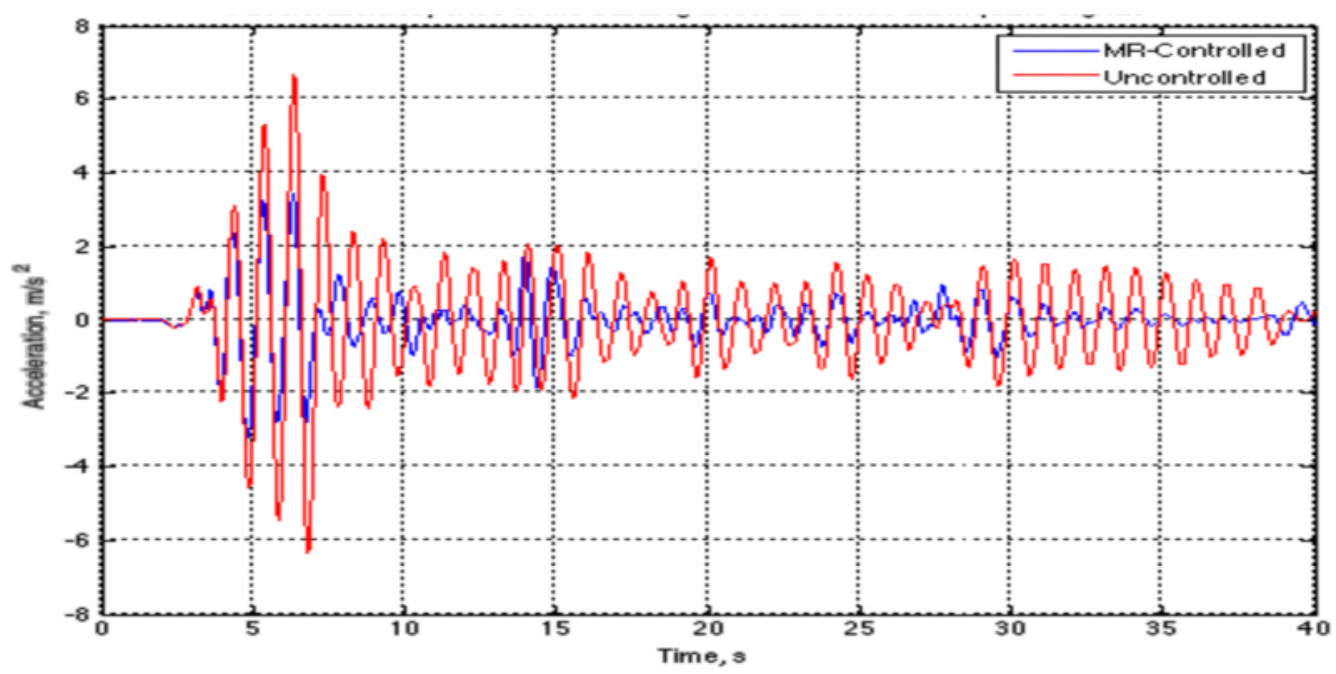

Fig. 6 Acceleration response. 


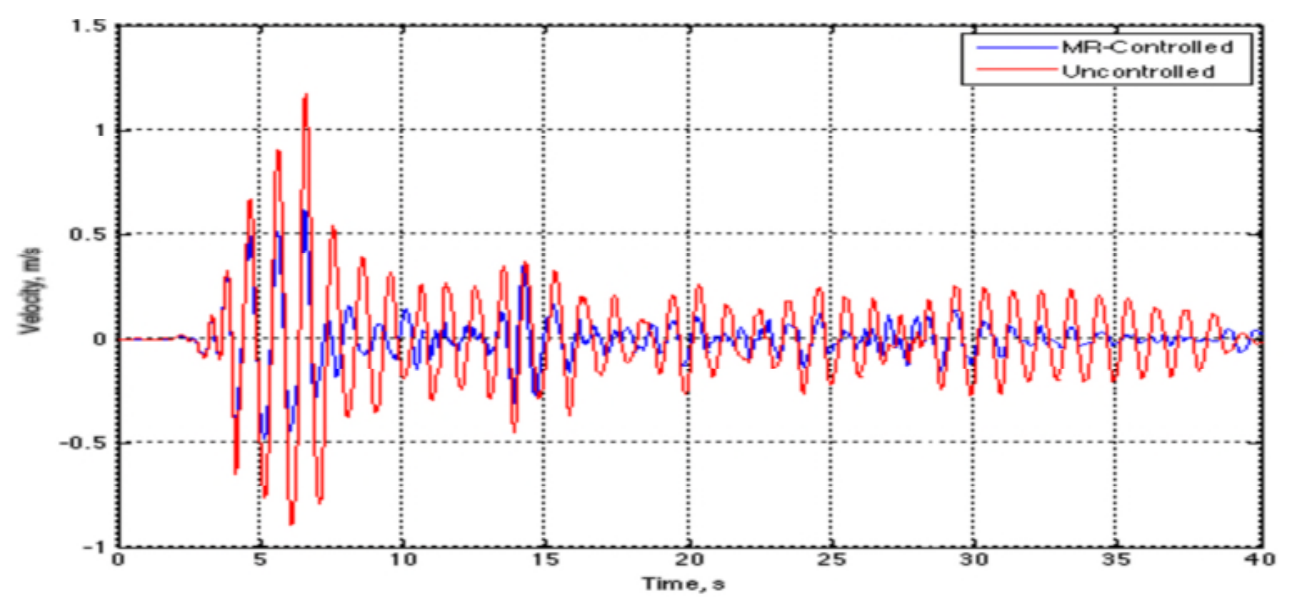

Fig. 7 Velocity response.

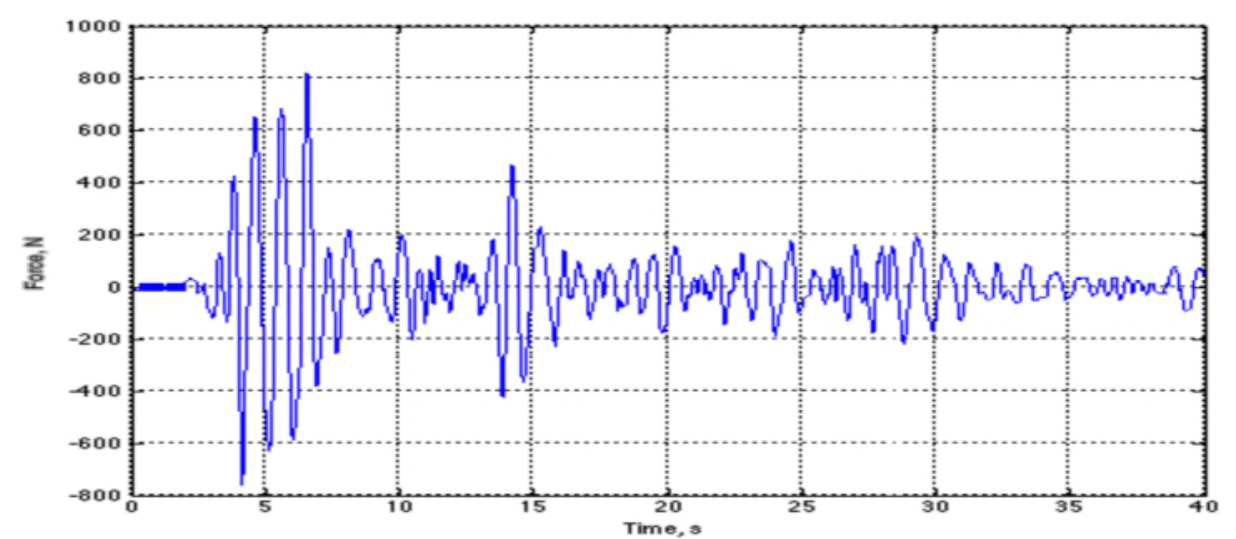

Fig. 8 Semi-active control Force.

TMD control of the initial displacement of the initial control is not obvious, the individual points beyond the control of the peak value, but the overall control. MR-TMD control similar to the control effect on the displacement of the structure with TMD Control effect. This is because the excitation caused by the displacement of the structure is not obvious, a variety of control strategies on the displacement control effect play a limited. The acceleration response of the structure is relatively strong, MR-TMD control effect on acceleration significantly better than TMD control.MR-TMD controlled speed, size control, but the direction is not always consistent with the main structure direction.

\section{Conclusions}

(1) On top of the traditional TMD control method on the basis of added mass creation of MR damper in the other layer, MR-TMD of semi-active control system of energy-absorbing capacities are increased. Study on the effect of TMD shock absorption shows that the damping of TMD shock absorption system not only with the TMD and the main structure of frequency ratio, but also with the TMD and the main structure of the quality ratio and damping of TMD system and is closely related to the position, while external excitation spectrum and composition.

(2) Reasonable choice of where the damper is installed, minimum number of dampers for optimum damping effect, so as to achieve economic goals. Dampers should be arranged in the location of inter laminar deformation to minimize torsion and stiffness, form a reasonable force.

(3) Minimize the differences of the center of mass and stiffness, install the damper should try to maintain the structure symmetrical, narrow the differences of the center of mass and stiffness. Because symmetric structures in earthquakes, General translational vibration, force more evenly and asymmetric structure of stiffness of deviation from Center, in earthquake prone to torsional vibration, stress is not uniform. 


\section{References}

[1] X. C. Zhu and X. J. Jing, Magnetorheological fluid dampers: A review on structure design and analysis, Intelligent Material Systems and Structures. SAGE, 23(8) (2012) 839-873.

[2] F. Gordaninejad, X. Wang and G. Hitchcock, Modular High-Force Seismic MagnetoRheological Fluid Damper, ASCE, 136 (2010) 135-143.

[3] J. D. Carlson, New cost Effective Braking Damping and Vibration Control Devices Made with Magnetorheological Fluidsc, Mater. Technol. 13 (1998) 96-99.

[4] L. L. Sui, MR-TMD structural damping vibration system parameters influence analysis, J. Shenzhen U. Sci. Eng. E. 23(4) (2006) 52-58.

[5] Code for seismic design of buildings in [1] (GB50011-2010), Beijing: China Building Industry Press, 2010.

[6] W. L. Qu, Intelligent control of intelligent materials and devices and structures for magnetorheological fluids, Beijing: Science Press, 2010. 